\title{
A prospective, randomized, double-blind, placebo-controlled trial of polyphenols on the outcomes of inflammatory factors and oxidative stress in patients with type 2 diabetes mellitus
}

\author{
Milkica Grabež ${ }^{1,2, *}$, Ranko Škrbić ${ }^{3}$, Miloš P Stojiljković ${ }^{3}$, Vesna Vučić ${ }^{4}$, \\ Vesna Rudić Grujić ${ }^{1,2}$, Vladimir Jakovljevićs ${ }^{5,6}$, Dragan M Djuric ${ }^{7}$, Relja Suručić ${ }^{8}$, \\ Katarina Šavikin ${ }^{9}$, Dubravka Bigović ${ }^{9}$, Nađa Vasiljević ${ }^{10}$ \\ ${ }^{1}$ Department of Hygiene, Faculty of Medicine University of Banja Luka, 78000 Banja Luka, the Republic of Srpska, Bosnia \& Herzegovina \\ ${ }^{2}$ Department of Hygiene, Public Health Institute of Republic of Srpska, 78000 Banja Luka, the Republic of Srpska, Bosnia \& Herzegovina \\ ${ }^{3}$ Department of Pharmacology, Toxicology and Clinical Pharmacology, Faculty of Medicine University of Banja Luka, 78000 Banja Luka, the Republic \\ of Srpska, Bosnia \& Herzegovina \\ ${ }^{4}$ Centre of Research Excellence in Nutrition and Metabolism, Institute for Medical Research University of Belgrade, 11000 Beograd, Serbia \\ ${ }^{5}$ Department of Physiology, Faculty of Medical Sciences University of Kragujevac, 34000 Kragujevac, Serbia \\ ${ }^{6}$ Department of Human Pathology, 1st Moscow Medical University "I.M. Sechenov", 119991 Moscow, Russian Federation \\ ${ }^{7}$ Institute of Medical Physiology "Richard Burian", Faculty of Medicine University of Belgrade, 11000 Belgrade, Serbia \\ ${ }^{8}$ Department of Pharmacognosy, Faculty of Medicine University of Banja Luka, 78000 Banja Luka, the Republic of Srpska, Bosnia \& Herzegovina \\ ${ }^{9}$ Institute for Medicinal Plant Research "Dr Josif Pančić", 11000 Belgrade, Serbia \\ ${ }^{10}$ Institute of Hygiene and Medical Ecology, Faculty of Medicine University of Belgrade, 11000 Belgrade, Serbia \\ *Correspondence: milkica.grabez@med.unibl.org (Milkica Grabež) \\ Academic Editor: Peter A. McCullough \\ Submitted: 14 October 2021 Revised: 10 December 2021 Accepted: 13 December 2021 Published: 11 February 2022
}

\begin{abstract}
Background: Type 2 diabetes mellitus (T2DM) is commonly associated with hyperglycemia, dyslipidemia, oxidative stress and inflammation which are well known cardiovascular risk factors. Pomegranate peel polyphenols have a proven hypolipemic, antioxidant and anti-inflammatory activity. However, there is a lack of clinical studies that would confirm its antioxidant and anti-inflammatory effects in diabetic patients. The potential of pomegranate peel extract (PoPEx) to counteract inflammation and oxidative stress in T2DM patients was investigated. For this purpose, a randomized, double-blind placebo-controlled study involving adult T2DM patients treated with PoPEx or placebo for eight-weeks was conducted. Methods: Patients were randomly divided into two groups: the first group (n =30) received capsules containing PoPEx $250 \mathrm{mg}$ twice daily, while the placebo group $(\mathrm{n}=30)$ received placebo capsules twice daily. Plasma concentration of inflammatory factors (interleukin 6 (IL-6), tumor necrosis factor $\alpha$ (TNF- $\alpha$ ) and high sensitivity C reactive protein (hsCRP)), oxidative stress biomarkers (thiobarbituric acid reactive substances (TBARS), nitrites $\left(\mathrm{NO}_{2}{ }^{-}\right.$), superoxide anion radical $\left(\mathrm{O}_{2}{ }^{-}\right)$, hydrogen peroxide $\left(\mathrm{H}_{2} \mathrm{O}_{2}\right)$, total antioxidant capacity (TAC)), homocysteine and lipid profile were analyzed. Results: The PoPEx treatment showed a significant reduction of inflammatory factors (IL-6, TNF- $\alpha$, hSCRP), oxidative stress biomarkers (TBARS, $\mathrm{NO}_{2}{ }^{-}, \mathrm{O}_{2}{ }^{-}$) and homocysteine, while the TAC was increased. Moreover, a significant improvement in lipid profile was observed in the PoPEx group. Additional analysis showed a significant inverse correlation between the decrements of all measured inflammatory markers and TAC in the PoPEx group. Conclusions: The study demonstrated that eight-week-long PoPEx administration had favorable effects on inflammatory status and oxidative stress biomarkers in diabetic patients.
\end{abstract}

Keywords: pomegranate peel extract; polyphenols; oxidative stress; inflammation; lipid profile; diabetes mellitus

\section{Introduction}

Type 2 diabetes mellitus (T2DM) makes $90-95 \%$ of all diabetes, affecting $6.28 \%$ of the world population globally $[1,2]$. The risk of T2DM increases with age, and it occurs more frequently in obese individuals and individuals with a sedentary lifestyle [1]. Hyperglycemia, dyslipidemia, oxidative stress and inflammation are involved in the pathogenesis of T2DM [3-5]. Thus, hyperglycemia can increase production of free radicals, which potentiates lipid peroxidation and protein nitration, activation of stresssensitive pathways and increased DNA damage [6]. Expo- sure of pancreatic $\beta$-cells to the oxidative stress may reduce insulin gene expression through the inhibition of promoter activity and insulin mRNA expression [7]. Furthermore, oxidative stress induced by hyperglycemia is believed to increase the level of pro-inflammatory proteins and cytokines [8]. The increased secretion of tumor necrosis factor $\alpha$ (TNF- $\alpha$ ) is linked with obesity-associated insulin resistance [9]. Adipose tissue, mostly visceral, represent the main source of pro-inflammatory mediators in T2DM such as TNF- $\alpha$, IL-1, IL-6, IL-10, leptin, adiponectin and other bioactive substances collectively called adipokines [10]. 
TNF- $\alpha$ and IL-6 can directly impair pancreatic $\beta$-cell function but also indirectly, by producing free fatty acids. These mechanisms promote the development of T2DM confirming the link between insulin resistance and inflammation. Previous research reports showed that inflammation and oxidative stress are associated with the risk of developing cardiovascular complication in T2DM patients [4]. Understanding the role of pro-inflammatory cytokines and inflammatory processes in the development of T2DM suggests that therapeutic approaches that can inhibit inflammation could be useful in the prevention and control of T2DM, as well as cardiovascular compliations in diabetic patients [4]. Considering a large number of people affected by obesity and obesity-related metabolic disorders, including T2DM worldwide, these therapeutic approaches can have a huge economic and public health impact.

Pomegranate (Punica granatum L.) is used in traditional medicine of different cultures. It is a rich source of polyphenols and other phytochemicals, including sterols, terpenoids, alkaloids and tocopherols, as compounds with important physiological actions $[11,12]$. These phytochemicals extracted from different parts of the fruit possess significant anti-diabetic, antioxidant and anti-inflammatory effects, as well as lipid lowering and antimi-crobial activity $[13,14]$. Pomegranate peel makes up to $40-50 \%$ weight of the fruit and due to its high polyphenol content possess strong antioxidant activity $[15,16]$. The pomegranate extracts have been used as a functional food for the years [1719]. The results of recently published studies have shown the beneficial effects of pomegranate peel extract (PoPEx) on body composition, systolic and diastolic blood pressure and lipid profile in overweight T2DM patients [20,21]. In addition, PoPEx has demonstrated no effect on fasting blood glucose level, but decreased the level of glycolisated hemoglobin after of 8 weeks' administration in diabetic patients. Although several biologically active polyphenols have been detected in the pomegranate peel, making it the richest source of tannins and flavonoids among all parts of the fruit, clinical studies are needed to confirm its antioxidant and anti-inflammatory effect in diabetic patients.

The aim of this study was to investigate the potential of PoPEx to attenuate the inflammation and oxidative stress in T2DM patients.

\section{Materials and methods}

\subsection{Study population and study design}

This randomized double-blind placebo-controlled study involved 60 overweight patients with T2DM. The patients were recruited at the Endocrinology Department of the University Clinical Centre of the Republic of Srpska, Banja Luka, Bosnia and Herzegovina, from June to November 2018. The study inclusion criteria were: $40-65$ years of age, BMI $\geq 25 \mathrm{~kg} / \mathrm{m}^{2}$, and glycosylated hemoglobin $(\mathrm{HbA} 1 \mathrm{C}) \geq 6.5 \%$. In addition, all patients had been receiving only oral hypoglycemic agent metformin for at least one year before the study enrolment and continued taking metformin during the whole study period. Patients with chronic kidney, liver, or inflammatory diseases and those taking hormone replacement therapy (insulin or other), antiinflammatory drugs, or antioxidant supplementation were not included in the study. The study protocol was explained and signed written informed consent was obtained from all the participants.

After assessing the National Diabetes Register 84 patients who met the inclusion criteria were identified and 60 patients accepted to participate in the study. Patients were randomly divided into 2 groups, by an impartial person, using 1:1 block randomization: the first group $(\mathrm{n}=$ 30) received capsules containing PoPEx $250 \mathrm{mg}$, twice a day (PoPEx group), while the placebo group $(n=30)$ received visually identical capsules containing placebo (250 $\mathrm{mg}$ ), twice a day. Both groups continued with regular diet during the 8 weeks' intervention period. The study was double blinded, meaning that no patients nor investigators were aware of which intervention is being administrated.

\subsection{Pomegranate peel}

Pomegranate (Punica granatum L.) was deposited in Botanical Garden "Jevremovac" University of Belgrade (woutcher specimen No BEOU 17742). Pomegranate fruits were picked and washed by hand in the east Herzegovina region (south of Bosnia and Herzegovina). The peel was separated from fruit and dried 4-6 days and grounded in powder. For the preparation of peel extract, ethanol was used as solvent. Phenolic compounds of PoPEx were quantified using high performance liquid chromatography (HPLC) methodology [21]. Quantification of ellagic acid, gallic acids, punicalagin and punicalin was done using calibration curves of authentic standards. The results are presented as milligrams per gram of dry weight (mg/g DW). The obtained dry extract contained punicalagin $69.67 \pm$ $0.72 \mathrm{mg} / \mathrm{g}$. Punicalin $30.41 \pm 0.11 \mathrm{mg} / \mathrm{g}$, ellagic acid 23.83 $\pm 0.72 \mathrm{mg} / \mathrm{g}$ and gallic acid $10.46 \pm 0.04 \mathrm{mg} / \mathrm{g}$. Flavonoids such as quercetin, rutin and kaempferol as well as cyanidin3-glycoside were also detected in extract. Total tannins were detected spec-trophotometrically according to European Pharmacopoeia 8.0 [22] and the amount was $11.8 \%$ which is in accordance with WHO monograph [23]. Each capsule contained $250 \mathrm{mg}$ of the pomegranate peel extract. To determine the amount of extract in capsules, the WHO monograph was used [23]. Placebo capsule contained only excipients, lactose, starch and magnesium stearate.

\subsection{Anthropometric measurements}

All parameters were measured at baseline and at the end of the study. Height was measured using wall-mounted stadiometer to the nearest $0.1 \mathrm{~cm}$. Body mass was measured using Tanita body composition analyzer. Body mass index (BMI) was calculated as weight $(\mathrm{kg})$ divided by square of height $\left(\mathrm{m}^{2}\right)$. 


\subsection{Energy intake}

All the patients filled out 3 days' prospective food records at baseline and at last 3 days of the intervention period to estimate energy intake and to follow potential changes during the study, that might affect the results. The energy intake was estimated according to the Serbian Food Composition Database, harmonized with EuroFIR standards end embedded in EuroFIR Food Platform and Balkan Food Platform [24].

\subsection{Biocemical analysis}

Blood samples were taken after 12-14 hours overnight fasting at beginning of the study, and following the intervention period. Serum lipid profile including triglycerides (TG), total cholesterol (TC), high-density lipoprotein cholesterol (HDL-C), low-density lipoprotein cholesterol (LDL-C), fasting glucose, glycosylated hemoglobin $\left(\mathrm{HbA}_{1} \mathrm{C}\right)$, and high-sensitivity C-reactive protein (hsCRP) were determined in serum, on the same day when the samples were collected, using the enzymatic methods on Roche Cobas 6000 (c501module) analyzer (Roche Diagnostics, Basel Switzerland) and colorimetric assay using commercially available kits, respectively. Triglyceride-glucose index (TyG) was calculated as follow: In [tryglicerides $(\mathrm{mg} / \mathrm{dL}) \times$ fasting glucose $(\mathrm{mg} / \mathrm{dL}) / 2]$. The plasma samples were stored at $-80{ }^{\circ} \mathrm{C}$ until the analysis of oxidative stress and inflammation parameters were done.

\subsection{Oxidative stress markers and homocysteine analyses}

Prooxidative parameters, index of lipid peroxidation, nitrites $\left(\mathrm{NO}_{2}{ }^{-}\right)$, superoxide anion radical $\left(\mathrm{O}_{2}{ }^{-}\right)$, and hydrogen peroxide $\left(\mathrm{H}_{2} \mathrm{O}_{2}\right)$ were determined in plasma samples using Shimadzu UV 1800 spectrophotometer (Japan). The index of lipid peroxidation, was assessed by an indirect method of determining of thiobarbituric acid reactive substances (TBARS) concentration by mixing $0.8 \mathrm{~mL}$ of plasma with $0.4 \mathrm{~mL} 1 \%$ thiobarbituric acid in $0.05 \mathrm{M}$ $\mathrm{NaOH}$. This method is based on the measurement of lipid peroxidase levels in the reaction of the malondialdehyde with thiobarbituric acid. Index of lipid peroxidation was measured in plasma samples at a wavelength of $530 \mathrm{~nm}$. For the blank tests, distilled water was used instead of plasma samples [25].

The nitrites $\left(\mathrm{NO}_{2}{ }^{-}\right)$in plasma samples were assessed by an indirect method since nitric oxide (NO), degrades rapidly to stable metabolites, such as nitrite/nitrate products. This method is based on the Griess reaction. Actually, $0.2 \mathrm{~mL}$ of plasma was mixed with $0.1 \mathrm{~mL}$ of $3 \mathrm{~N}$ perchloric acid, and $0.4 \mathrm{~mL}$ of $20 \mathrm{mM}$ ethylenediaminetetraacetic acid (EDTA). The mixture was put on ice for $15 \mathrm{~min}$, then centrifuged $15 \mathrm{~min}$ at $6000 \mathrm{rpm}$. After pouring off the supernatant, $220 \mu \mathrm{L} \mathrm{K}_{2} \mathrm{CO}_{3}$ was added. Nitrites were measured at a wavelength of $550 \mathrm{~nm}$. Distilled water was used as a blind test instead of plasma [26].
The determination of superoxide anion radical is based on the reaction of $\mathrm{O}_{2}{ }^{-}$with nitro blue tetrazolium to nitro formazan blue. In $50 \mu \mathrm{L}$ of plasma samples, $950 \mu \mathrm{L}$ of assay mixture ex tempore was added and measured three times every 60 seconds at a wavelength of $550 \mathrm{~nm}$. Instead of plasma samples, an appropriate amount of distilled water was used as a blank test [27].

For measuerement of $\mathrm{H}_{2} \mathrm{O}_{2}$ levels, oxidation of phenol red with hydrogen peroxide was applied, in a reaction catalyzed by enzyme horseradish peroxidase (HRPO). Red phenol solution $(800 \mu \mathrm{L})$ and peroxidase $(10 \mu \mathrm{L})$ were added to $200 \mu \mathrm{L}$ of plasma samples. After 10 minutes of incubation at room temperature, the $\mathrm{H}_{2} \mathrm{O}_{2}$ concentration was measured at wavelength of $610 \mathrm{~nm}$. Instead of plasma, distilled water was used for the blank test [28].

The homocysteine concentration was measured in serum by means of HPLC procedure with reverse-phase separation and fluorescence detection, as previously described. The fluorescence was determined spectrophotometrically at $390 \mathrm{~nm}$, while emission was measured at 470 $\mathrm{nm}$ [29].

The TAC was assessed by commercially available Sigma-Aldrich total antioxidant assay kit.

\subsection{Pro-inflammatory cytokines (IL-6, TNF- $\alpha$ )}

Plasma cytokine concentrations were determined in triplicates using human cytokine-specific ELISA assays (Human IL-6 DUOSET ELISA Development kit, R \&amp; D Systems, USA; Human TNF- $\alpha$ /TNFSF1A DUOSET ELISA Development kit, R \&amp; D Systems, USA) as recommended by the manufacturer. After addition of capture antibody $(100 \mu \mathrm{L})$ to the wells of micro titer plates (MTP), the plates were left overnight at room temperature. After adding the block buffer to the final volume of $300 \mu \mathrm{L}$, MTPs were incubated at room temperature for one hour. Diluted samples and standards were added to the wells and, incubated for additionaly 2 hours at room temperature. Then, $100 \mu \mathrm{L}$ of detection antibody was added to each well, and the plates were incubated for 2 hours at room temperature. In the next step, MTPs were washed and $100 \mu \mathrm{L}$ of horseradish streptavidin peroxidase was added to each well. Incubation was stopped after $20 \mathrm{~min}$ and $100 \mu \mathrm{L}$ of substrate solution (Color reagent $\mathrm{A}+$ Color reagent $\mathrm{B}, 1: 1$ ) was added. $50 \mathrm{~mL}$ of stop solution $\left(2 \mathrm{~N} \mathrm{H}_{2} \mathrm{SO}_{4}\right)$ was added after another 20 minutes incubation, and the absorbance, in each well, was directly measured at $450 \mathrm{~nm}$ using a Microplate Reader (Zenyth, Anthos, United Kingdom). The final apsorbance was calculated as measured values, reduced for the values of the blanks (deionized water). A standard curve was created, based on the absorbances of the standards [25].

\subsection{Statistical analysis}

The data were analyzed using the SPSS software (Version 20; IBM Chicago, IL, USA). The distribution of variables, was assessed by Shapiro-Wilk's test. Baseline val- 
Table 1. Patients' demographics and baseline clinical characteristics.

\begin{tabular}{lccc}
\hline Parameters & PoPEX group $(\mathrm{N}=30)$ & Placebo group $(\mathrm{N}=30)$ & $p$ value \\
\hline Female N $(\%)$ & $15(50.00)$ & $15(50.00)$ & 1.000 \\
Age $($ years, mean $\pm \mathrm{SD})$ & $57.87 \pm 6.08$ & $56.93 \pm 6.67$ & 0.573 \\
$\mathrm{BMI}\left(\mathrm{kg} / \mathrm{m}^{2}\right.$, mean $\left.\pm \mathrm{SD}\right)$ & $30.95 \pm 4.37$ & $31.73 \pm 4.89$ & 0.512 \\
$\mathrm{HbA}$ C $(\%$, mean $\pm \mathrm{SD})$ & $7.55 \pm 1.22$ & $7.67 \pm 1.36$ & 0.882 \\
Duration of DM (months, mean $\pm \mathrm{SD})$ & $56.25 \pm 38.03$ & $64.09 \pm 49.78$ & 0.941 \\
Age at onset of DM (years, mean $\pm \mathrm{SD})$ & $51.93 \pm 6.82$ & $50.77 \pm 7.23$ & 0.523 \\
Current smoking status N $(\%)$ & $10(33.33)$ & $5(17.86)$ & 0.179 \\
\hline
\end{tabular}

PoPEx, Pomegranate peel extract; BMI, Body mass index; $\mathrm{HbA}_{1} \mathrm{C}$, Glycosylated hemoglobin; DM,

Diabetes mellitus. For comparisons between groups at baseline, Chi square test, Student's $t$ test and

Mann-Whitney U test were used.

ues were computed by Chi square test, Student's $t$ test and Mann-Whitney $U$ test depending on the data distribution. For analysis of differences in the outcome variables at the end of study compared to the baseline values, paired sample $t$-test or Wilcoxon Signed Rang test were conducted. For assessing differences between the two groups we used mixed-model analysis of variance (mixed ANOVA), and non-parametric alternative to mixed ANOVA Bruner and Langer test. Correlations between delta values of inflammatory markers and oxidative stress biomarkers were calculated using Spearman's rank correlation. Differences in $p$ value of $p<0.05$ were considered significant.

\section{Results}

Sixty patients with T2DM were enrolled in the study, but two of them from the placebo group were excluded from further analysis due to discontinuation of the therapy. The baseline characteristics of the PoPEx and the placebo groups are displayed in Table 1. No differences between the two groups in the age and gender of study participants, serum level of $\mathrm{HbA}_{1} \mathrm{C}$, duration of T2DM and metformin therapy were found. The results of 3 days' food record showed no significant changes between groups in mean energy and micronutrient intake during the study [20]. In both study groups, no adverse effects were reported during the follow-up period.

After 8 weeks' of PoPEx supplementation we noticed significantly lowered serum values of $\mathrm{HbA}_{1} \mathrm{C}$ in $\mathrm{PoPEx}$ group $(7.55 \pm 1.22$ vs. $7.32 \pm 1.02 \%, p<0.001)$, while the values remained unchanged in the placebo group.

There were no differences in parameters of oxidative stress at baseline between the two groups. However, after eight weeks of intervention, all oxidative stress markers (TBARS, $\mathrm{NO}^{-}, \mathrm{O}_{2}{ }^{-}$and homocysteine) except $\mathrm{H}_{2} \mathrm{O}_{2}$ decreased significantly in the PoPEx group, while the TAC significantly increased. At the same time, no differences were detected in the placebo group except the TAC, which also increased. Thus, markedly lower values of oxidative stress markers (TBARS, $\mathrm{NO}^{-}, \mathrm{O}_{2}{ }^{-}$) were found in the PoPEx group than in the placebo group at the end of the study. The results were similar after conducted mixed ANOVA, except for the TAC value (Table 2).

No significant differences were found in the lipid profiles between the PoPEx group and the placebo group at the beginning of study. However, the eight weeks' consumption of PoPEx capsules had a significant influence on lipid profile. As shown in Table 3, a significant decrease in the levels of TC, LDL-C and TG, associated with significant increase in HDL-C, were noticed in the PoPEx group, compared with baseline values. No significant changes were observed in the placebo group. Furthermore, analysis of between group changes showed significant differences in the levels of TG and HDL-C. A part of these results related to lipid dynamics have been already published [21].

The eight weeks of PoPEx supplementation resulted in significant decrease of the inflammatory parameters, compared to baseline values (hsCRP: median, Interquartile Range (IQR) $2.05 \mathrm{mg} / \mathrm{L}, 1.18-3.17$ vs. $1.75 \mathrm{mg} / \mathrm{L}$, 0.97-2.67; IL-6: $139.50 \mu \mathrm{mol} / \mathrm{L}, 94.04-199.95$ vs. 123.50 $\mu \mathrm{mol} / \mathrm{L}, 77.29-168.75$, and TNF $\alpha 172.80 \mathrm{pg} / \mathrm{L} 140.30$ 233.10 vs. 139.00 pg/L, 104.91-189.83) (Fig. 1A-C). At the same time, serum levels of hsCRP, IL- 6 and TNF- $\alpha$ were higher at the end of he study in placebo group. The differences between the basal and final values (dellta values) of inflammatory parameters were significantly different between the two groups after the intervention period (Fig. 1DF).

Finally, the correlations between changes in oxidative stress and inflammatory markers were tested. Significant inverse correlations were found between TAC and all measured markers of inflammation: $\Delta$ IL-6, $\Delta$ TNF- $\alpha$, and $\Delta$ hsCRP. In addition, $\Delta$ IL-6 inversely correlated with $\Delta$ $\mathrm{H}_{2} \mathrm{O}_{2}$ and positively with $\Delta \mathrm{NO}^{-}$(Table 4).

\section{Discussion}

A growing interest in polyphenol research was noticed in the last 20 years. Although the current evidence is mostly based on the results of observational and in vitro studies, which have elucidated the potential mechanisms of the protective effects of polyphenols. There are a meager number 
Table 2. The effects of eight-week PoPEx consumption on homocysteine and oxidative stress parameters in patients with T2DM.

\begin{tabular}{lccccc}
\hline \multirow{2}{*}{ Oxidative stress parameters } & \multicolumn{2}{c}{ PoPEx group } & \multicolumn{2}{c}{ Placebo group } & \multirow{2}{*}{$p$ value } \\
\cline { 2 - 5 } & Before treatment & After treatment & Before treatment & After treatment & \\
\hline Homocysteine $\mathrm{mmo} / \mathrm{L}$ & $9.63 \pm 1.59$ & $8.74 \pm 0.098^{* * *}$ & $8.30 \pm 1.19$ & $8.56 \pm 1.27$ & 0.037 \\
$\mathrm{TBARS} \mu \mathrm{mol} / \mathrm{mL}$ & $1.60 \pm 0.29$ & $0.38 \pm 0.15^{* * *}$ & $1.56 \pm 0.17$ & $1.47 \pm 0.23$ & $<0.001$ \\
$\mathrm{O}_{2}{ }^{-} \mathrm{nmol} / \mathrm{mL}$ & $5.81 \pm 1.09$ & $3.19 \pm 1.40^{* * *}$ & $5.54 \pm 1.36$ & $4.84 \pm 1.75$ & $<0.001$ \\
$\mathrm{NO}_{2}{ }^{-} \mathrm{nmol} / \mathrm{mL}$ & $14.04 \pm 2.17$ & $6.95 \pm 1.94 * * *$ & $14.59 \pm 1.87$ & $15.54 \pm 2.28$ & $<0.001$ \\
$\mathrm{H}_{2} \mathrm{O}_{2} \mathrm{nmol} / \mathrm{mL}$ & $2.69 \pm 0.28$ & $2.99 \pm 0.37$ & $3.00 \pm 1.20$ & $3.11 \pm 0.36$ & 0.504 \\
$\mathrm{TAC} \mu \mathrm{mol} / \mathrm{L}$ & $149.93 \pm 69.00$ & $230.81 \pm 84.72^{* *}$ & $165.50 \pm 83.35$ & $205.64 \pm 83.68^{*}$ & 0.154 \\
\hline
\end{tabular}

Data are expressed as the mean \pm SD. PoPEx, pomegranate peel extract; T2DM, type 2 diabetes mellitus; TBARS, thiobarbituric acid reactive substances; TAC, total antioxidant capacity. Paired sample $t$-tests were used to test for changes over time within the the groups and done and asterisk $\left(^{*}\right)$ indicate significant differences compared with baseline value $* p<0.05, * * p<0.01$ and $* * * p<0.001$. Mixed ANOVA interaction effects were used to test for differences between the groups after controlling for the baseline values ( $p$-value).

Table 3. The effects of eight-week PoPEx consumption on lipid profile in patients with T2DM.

\begin{tabular}{lccccc}
\hline \multirow{2}{*}{ Lipid profile } & \multicolumn{2}{c}{ PoPEx group } & \multicolumn{2}{c}{ Placebo group } & \multirow{2}{*}{$p$ value } \\
\cline { 2 - 4 } & Before treatment & After treatment & Before treatment & After treatment & \\
\hline TC mmol/L & $5.46 \pm 1.36$ & $5.13 \pm 1.29^{*}$ & $5.22 \pm 0.76$ & $5.27 \pm 0.67$ & 0.138 \\
LDL-C mmol/L & $3.62 \pm 1.13$ & $3.32 \pm 1.11^{*}$ & $3.53 \pm 0.79$ & $3.52 \pm 0.72$ & $<0.186$ \\
TG mmol/L & $2.83 \pm 2.13$ & $2.20 \pm 1.87^{* * *}$ & $2.27 \pm 1.24$ & $2.34 \pm 1.53$ & $<0.001$ \\
HDL-C mmol/L & $1.11 \pm 0.21$ & $1.34 \pm 0.21^{* * *}$ & $1.06 \pm 0.24$ & $1.10 \pm 0.28$ & $<0.001$ \\
TyG & $9.69 \pm 0.63$ & $9.44 \pm 0.65^{* * *}$ & $9.52 \pm 0.56$ & $9.63 \pm 0.59$ & $<0.001$ \\
\hline
\end{tabular}

Data are expressed as the mean \pm SD; PoPEx, pomegranate peel extract; T2DM, type 2 diabetes mellitus; TC, total cholesterol; LDL-C, low-density lipoprotein cholesterol; TG, triglycerides; HDL-C, high-density lipoprotein cholesterol; TyG, Trygliceride/glucose index. Paired sample $t$-tests were used to test for changes over time whithin the groups and asterisk $(*)$ indicate significant differences compared with baseline value ${ }^{*} p<0.05$. Mixed ANOVA interaction were used to test for differences between the groups after controlling for the baseline values ( $p$-value). Due to outlieres evident in TG and HDL-C, for these outcomes we used Wilcoxon signed rank test was done and asterisk $(*)$ indicate significant differences compared with baseline value $* p<0.05,{ }^{* * *} p<0.001$. Also, for these outcomes we used Brunner and Langer non-parametric alternative to mixed ANOVA.

of clinical studies that clarify the effects of pomegranate [30]. Within these, most studies have been conducted using pomegranate juice or whole fruit extracts. The doses, study design and the duration of these studies varied from study to study [13].

The interaction of oxidative stress and low-grade inflammation has been reported as a known pathway in the development and progression of diabetes mellitus and its complications, including CVD [4]. Lipid metabolism and peroxidation are important for development of inflammation which is elevated in several diabetic complications [31]. This randomized, placebo-controlled intervention study lasting eight weeks is, to the best of our knowledge, among the first studies that described the effects of pomegranate peel extract on the oxidative state and inflammation in T2DM patients. We found that consumption of a standardized PoPEx, used in this study, significantly influenced the markers of oxidative stress, inflammation parameters and lipid profile in diabetic patients.
Additionally, as we previously reported, consumption of PoPEx significantly lowered serum values of $\mathrm{HbA}_{1} \mathrm{C}$ in PoPEx group, while fasting blood glucose was unaffected [21]. Several studies show that the inadequate glycemic control indicated higher risk of diabetes-related complication and death [32]. Better glucoregulation and, in the same time antioxidant and anti-inflammatory activities of PoPEx contribute to better diabetes management [33].

Homocysteine, a sulphur containing amino acid is an independent risk factor for cardiovascular disorders [29]. It may induce mitochondrial dysfunction trough an increase in reactive oxygen species (ROS) production [34] and promote oxidative injury to vascular cells [35]. A previous study suggested that synergistic effects of moderately elevated levels of homocysteine and oxidative stress markers in diabetes promote the development of atherosclerosis [35]. However, homocysteine levels in diabetic patients are controversial. Numerous studies reported elevated level of homocysteine [36,37], but some other studies found un- 


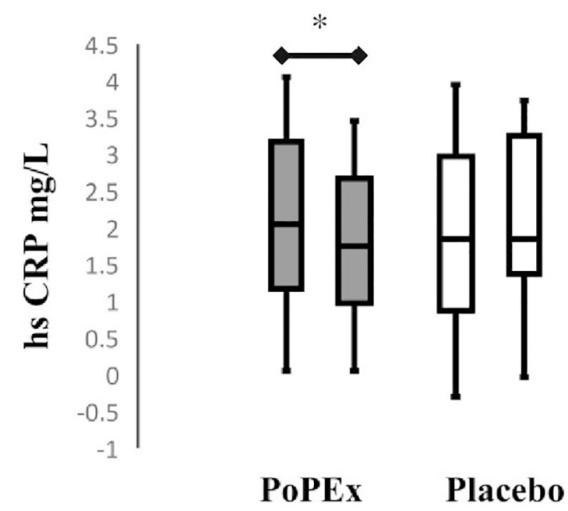

(B)

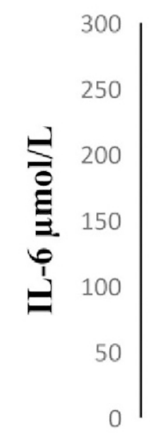

(C)

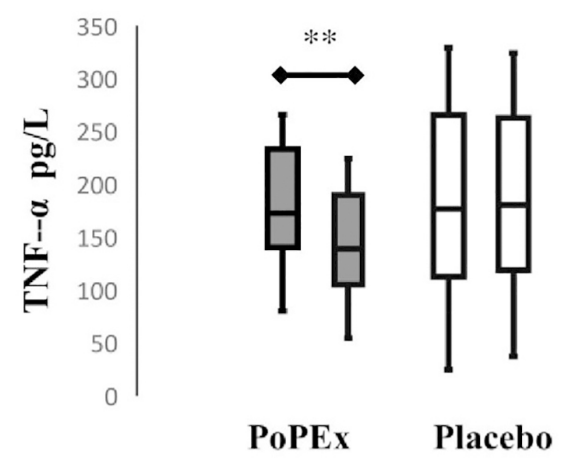

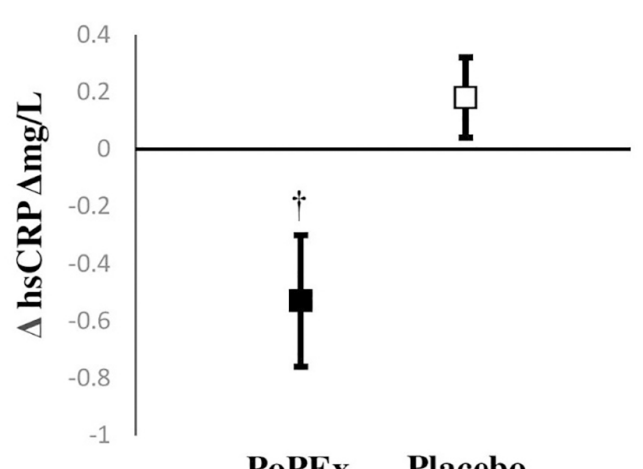

PoPEx Placebo

(E)

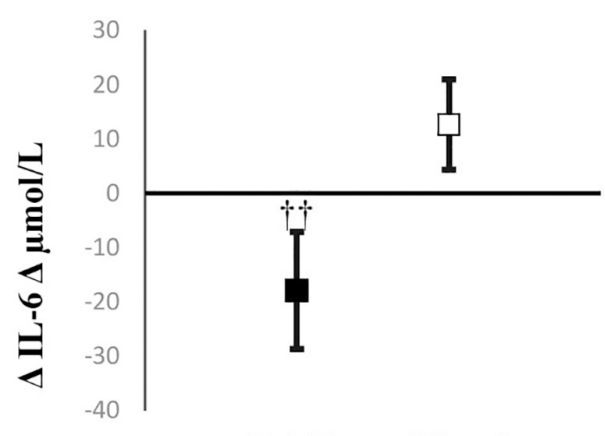

(F)

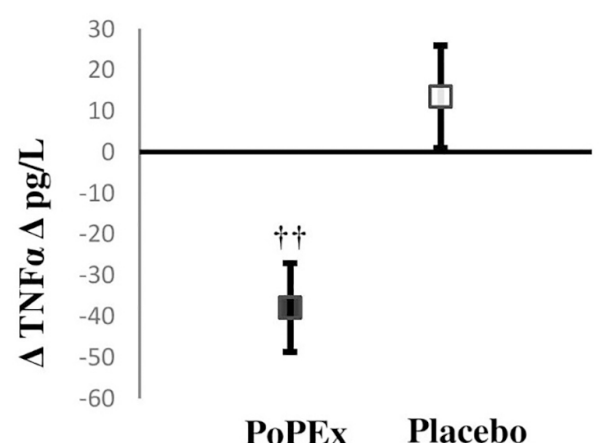

Fig. 1. The effects of eight-week PoPEx consumption on the circulating biomarkers of inflammation levels. (A-C) The high sensitivity c-reactive protein (hsCRP), Interleukin 6 (IL-6), and tumor necrosis factor $\alpha$ (TNF- $\alpha$ ) levels, baseline and after eight-weeks intervention period in the Diabetes mellitus type 2 patients. Wilcoxon signed rank test was done and asterisk $(*)$ indicate significant differences compared with baseline value within the groups $* p<0.05,{ }^{* *} p<0.01$. (D-F) The delta values of circulating inflammation biomarkers levels (hsCRP, IL-6, and TNF- $\alpha$ ) in the PoPEx group and the Placebo group. Mixed ANOVA was done and sign ( $\dagger$ ) indicate significant differences between the groups after controlling for the baseline values $\uparrow p<0.05, \uparrow \uparrow p<0.01$.

changed [38] and even reduced homocysteine level in these patients [39]. At the beginning of the present study the level of homocysteine was within the normal range, but after eight weeks of PoPEx treatment the serum level of homocysteine was significant decreased. Lowering homocys- teine level by $3 \mu \mathrm{mol} / \mathrm{L}$ is associated with a decrease of $16 \%$ in risk of ischemic heart disease and $24 \%$ in risk of stroke [40].

The results of this study demonstrated that PoPEx supplementation significantly reduced oxidative stress markers 
Table 4. Correlation between changes of inflammatory factors and oxidative stress markers in patients with T2DM.

\begin{tabular}{|c|c|c|c|c|c|}
\hline \multirow{2}{*}{ Group } & $\Delta$ TBARS mmol/L & $\Delta \mathrm{O}_{2}{ }^{-} \mu \mathrm{mol} / \mathrm{L}$ & $\Delta \mathrm{NO}^{-} \mathrm{mmol} / \mathrm{L}$ & $\Delta \mathrm{H}_{2} \mathrm{O}_{2} \mathrm{mmol} / \mathrm{L}$ & $\Delta \mathrm{TAC} \mu \mathrm{mol} / \mathrm{L}$ \\
\hline & $r / p$ & $r / p$ & $r / p$ & $r / p$ & $r / p$ \\
\hline \multicolumn{6}{|l|}{ PoPEx } \\
\hline$\Delta \mathrm{IL}-6 \mu \mathrm{mol} / \mathrm{L}$ & $0.108 / 0.577$ & $-0.032 / 0.868$ & $0.483 / 0.008$ & $-0.494 / 0.006$ & $-0.552 / 0.002$ \\
\hline$\Delta \mathrm{TNF}-\alpha \mathrm{pg} / \mathrm{L}$ & $0.064 / 0.740$ & $0.250 / 0.192$ & $0.339 / 0.072$ & $-0.368 / 0.050$ & $-0.399 / 0.029$ \\
\hline$\Delta \mathrm{hsCRP} \mathrm{mgl} / \mathrm{L}$ & $-0.159 / 0.409$ & $-0.104 / 0.591$ & $-0.242 / 0.206$ & $0.116 / 0.550$ & $-0.387 / 0.034$ \\
\hline \multicolumn{6}{|l|}{ Placebo } \\
\hline$\Delta \mathrm{IL}-6 \mu \mathrm{mol} / \mathrm{L}$ & $-0.205 / 0.295$ & $-0.332 / 0.084$ & $0.167 / 0.397$ & $-0.184 / 0.348$ & $0.156 / 0.438$ \\
\hline$\Delta \mathrm{TNF}-\alpha \mathrm{pg} / \mathrm{L}$ & $0.300 / 0.120$ & $0.184 / 0.297$ & $-0.109 / 0.582$ & $0.034 / 0.864$ & $0.341 / 0.082$ \\
\hline$\Delta \mathrm{hsCRP} \mathrm{mgl} / \mathrm{L}$ & $-0.139 / 0.481$ & $0.005 / 0.979$ & $0.012 / 0.950$ & $-0.165 / 0.402$ & $0.137 / 0.497$ \\
\hline
\end{tabular}

IL-6, Interleukin 6; TNF- $\alpha$, tumor necrosis factor alpha; hsCRP, high sensitivity c-reactive protein; TBARS, thio-

barbituric acid reactive substances; TAC, total antioxidant capacity. Spearman's rank correlation was used.

such as TBARS, $\mathrm{NO}^{-}$and $\mathrm{O}_{2}^{-}$and increase the TAC in T2DM patients. Previous studies reported that flavonoids from pomegranate juice a responsible for the improvement in antioxidant capacity and also the beneficial effect in limiting oxidative damage $[41,42]$. Furthermore, these findings are supported by the results in women with polycystic ovarian syndrome and diabetic patients $[43,44]$. Studies also reported an increase of NO serum level in T2DM patients $[45,46]$. Hyperglycemia as a common feature in T2DM, and duration of disease may enhance the NO production [45]. The higher level of NO combined with increased production of $\mathrm{O}_{2}{ }^{-}$in formation of peroxynitrate extremely reactive form of ROS, which damages proteins, lipids, and nucleic acids and promote LDL-oxidation and atherosclerotic plaque development, which further lead to cardiovascular complications $[8,47]$. The levels of these harmful compounds are significantly reduced after PoPEx intervention (Table 2). Possible mechanisms for improving oxidative stress following eight weeks of PoPEx supplementation include free radical scavenging and supporting antioxidant system $[14,48]$. Although several studies failed to show alleviation of cardiovascular complication using antioxidant supplementation (Vitamin E) [49,50] positive effects of polyphenols are more prominent [14,51]. However, there is a great variability and seasonal variations in polyphenols consumption between countries. The largest antioxidant contribution achieved through food consumption, was obtained from vegetables and fruits intake [52]. In population living in the four season zones the higher consumption of fruits and vegetables was observed in spring and summer [53]. This dietary intake pattern could explain the increased TAC noticed in the placebo group.

Our findings confirmed previously published results that an eight-week consumption of PoPEx induces statistically significant lipid profile improvement in patients with T2DM, compared to the placebo group [21]. We have confirmed a significant reduction in the plasma levels of $\mathrm{TG}$, TC and LDL-C, and an increase in HDL-C in PoPEx group (Table 3). The study conducted by Hosseini et al. [54], re- ported that a four-week-long administration of the concentrated extract of whole pomegranate induced a significant improvement in lipid profiles in obese people. Also, these outcomes are in accordance with previous studies [55-59], which used pomegranate juice or pomegranate seed oil supplementation. On the other hand, some authors reported that administration of pomegranate polyphenols did not resulted in any improvement in lipid profile [60,61]. The inconsistency of these results can be explained by several reasons: small sample size, differences in the applied doses, supplementation periods, and form of pomegranate products [62]. Previously, in vivo and in vitro studies suggested that pomegranate extracts had regulatory effects on lipid metabolism in human and animal adipose tissue. The eight-week administration of the hydromethanol peel extract in diabetic rats showed hypolipemic activities that was attributed to the powerful reactive oxygen scavenging properties of these compounds [19]. The inhibition of lipid synthesis and the inhibition of lipolysis are the mechanisms by which some intervention prevents ectopic lipid storage and insulin resistance. The molecular mechanism may involve the activation of peroxisome proliferator-activated receptor $\gamma(\operatorname{PPAR} \gamma)$ and the increased metabolism of cholesterol in LO2 cells [63], or decreased expression of sterol regulatory binding protein-1c (SREBP-1c) [64]. The main components of PoPEx, punicalagin and ellagic acid, have lipidlowering effect in a dose-dependent manner. Disturbances of lipid metabolism in T2DM patients may lead to overproduction of lipid peroxidation products which may cause elevation of oxidative stress [65].

Elevated TyG index as a reliable marker of insulin resistance, and it is associated with progression of coronary artery calcification reflecting cardiovascular risk [66]. Also, TyG index was strongly associated with cardiovascular events in patients with T2DM [67]. As it can be seen in the Table 3, PoPEx significantly reduced this index, suggesting the potential effect of POPEx in reducing risk for cardiovascular complications in T2DM patients. 
The increased levels of inflammatory biomarkers such as IL-6, TNF- $\alpha$ and hsCRP observed in T2DM have been widely reported [68]. It is known that raised levels of IL-6, and TNF- $\alpha$ can promote insulin resistance and contribute to the development of T2DM [69,70]. Furthermore, hsCRP as a mediator of atherosclerotic diseases is strongly associated with the cardiovascular disease risk in T2DM patients [71].

In the present study, a reduction of plasma concentration of inflammatory parameters IL-6, TNF- $\alpha$ and hsCRP $(21 \%, 12 \%$ and $20 \%$, respectively) following the eightweek consumption of PoPEx in diabetic patients was observed. In line with our results, the anti-inflammatory effects of pomegranate or its derivatives had been previously shown in several studies. Significant decreases in IL-6 and hsCRP concentrations (by 30\% and 32\%, respectively) were observed after twelve-week administration of $250 \mathrm{~mL} /$ day pomegranate juice in diabetic patients [72]. Boldaji and coworkers demonstrated beneficial effects of eight-week consumption of $100 \mathrm{~mL}$ pomegranate juice in hemodialysis patients by reduction of CRP and IL-6 [73]. In an experimental models of rheumatoid arthritis administration $200 \mathrm{mg} / \mathrm{kg}$ of pomegranate rind extract significantly ameliorate inflammation parameters such as TNF$\alpha$, IL-6 and nuclear factor kappa B (NF- $\kappa \mathrm{B})$ suggesting that inhibition of NF- $\kappa \mathrm{B}$ could be responsible for its antiinflammatory effect [74]. Mastrogiovanni et al. [75] suggested that punicalagin as bioactive component could be related to anti-inflammatory activity of PoPEx trough inhibition of prostaglandin production. These results are supported by the results of a recent study that showed that punicalagin and ellagic acid, as main components of pomegranate peel, possess a potent antioxidative and antiinflammatory effect [76]. However, biological effects of polyphenols in vivo depend on their metabolism and this aspect will be addressed in a separate study.

The proposed mechanism of anti-inflammatory action of pomegranate is a decrease of the expression of cyclooxygenase- $2(\mathrm{COX}-2)$ via inhibition of the NF- $\kappa \mathrm{B}$ and mitogen activated protein kinase (MAPK) pathways reducing the generation of pro-inflammatory prostaglandins $[77,78]$. This is in accordance with our recent results on the fatty acid composition of plasma lipids of T2DM patients that the consumption of PoPEx inhibited conversion of arachidonic acid to pro-inflammatory eicosanoids and this could be one of possible anti-inflammatory mechanism of PoPEx activity [21].

The strenght of this study is in the use of standardized PoPEx polyphenols in T2DM patients with strong exclusion/inclusion criteria. The major limitations are that this is a small-scale study that is performed in one clinic, and that the study lacked the assessment of dietary polyphenol intake. Further larger studies should be conducted in order to document solid conclusions on whether a dietary component can improve health parameters in T2DM patients.

\section{Conclusions}

The study demonstrated that eight-week-long PoPEx administration had favorable effects on inflammatory status and oxidative stress biomarkers in diabetic patients. At the same time, the lipid profile improved significantly. Consumption of PoPEx could be advocated to be used as a functional food ingredient, considered as a dietary and natural remedy with pharmacological properties for prevention and treatment of diabetic patients.

\section{Author contributions}

MG, RS and MPS - contributed to the conception and design of the study; MG and VRG - collected data; $\mathrm{MG}$, VV, VJ, DMD — analysed the data; MG, RS , MPS, VV,

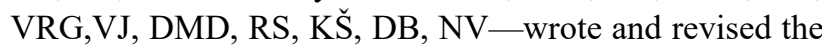
manuscript. All authors have read and agreed to the published version of the manuscript.

\section{Ethics approval and consent to participate}

The study was conducted according to the guidelines of the Declaration of Helsinki, and approved by the Ethics Committee of the Faculty of Medicine University of Banja Luka (No 01-9-604-2/17), the Republic of Srpska, Bosnia and Herzegovina, and by Ethics Committee o the University Clinical Centre of the Republic of Srpska (No18/4.37/17), Bosmia and Herzegovina. The study protocol was explained and written informed consent was obtained from all the participants involved in the study.

\section{Acknowledgment}

The authors would like to thank all the participants in the present study. The authors express appreciation to the Institute for Medicinal Plant Research "Dr Josif Pančić", Belgrade, Serbia for preparing the pomegranate peel extract.

\section{Funding}

This research was supported by the Ministry for Scientific-Technological Development, Higher Education and Information Society (No 19/6-020/961-81/18) Government of the Republic of Srpska.

\section{Conflict of interest}

The authors declare no conflict of interest. Vladimir Jakovljević and Dragan M Djuric are serving as Guest editors of this journal. We declare that Vladimir Jakovljević and Dragan M Djuric had no involvement in the peer review of this article and has no access to information regarding its peer review. Full responsibility for the editorial process for this article was delegated to Peter A. McCullough. 


\section{References}

[1] American Diabetes Association. 2. Classification and Diagnosis of Diabetes: Standards of Medical Care in Diabetes - 2021. Diabetes Care. 2021; 44: S15-S33.

[2] Khan MAB, Hashim MJ, King JK, Govender RD, Mustafa H, Al Kaabi J. Epidemiology of Type 2 Diabetes - Global Burden of Disease and Forecasted Trends. Journal of Epidemiology Global Health. 2020; 10: 107-111.

[3] Halim M, Halim A. The effects of inflammation, aging and oxidative stress on the pathogenesis of diabetes mellitus (type 2 diabetes). Diabetes \& Metabolic Syndrome: Clinical Research \& Reviews. 2019; 13: 1165-1172.

[4] Oguntibeju OO. Type 2 diabetes mellitus, oxidative stress and inlammation: examing the linx. International Journal of Physiology, Pathophysiology and Pharmacology. 2019; 11: 45-63.

[5] Parhofer KG. Interaction between Glucose and Lipid Metabolism: more than Diabetic Dyslipidemia. Diabetes \& Metabolism Journal. 2015; 39: 353-362.

[6] Goodarzi MT, Navidi AA, Rezaei M, Babahmadi-Rezaei H. Oxidative damage to DNA and lipids: correlation with protein glycation in patients with type 1 diabetes. Journal of Clinical Laboratory Analysis. 2010; 24: 72-76.

[7] Kawahito S, Kitahata H, Oshita S. Problems associated with glucose toxicity: Role of hyperglycemia-induced oxidative stress. World Journal of Gastroenterology. 2009; 15: 4137-4142.

[8] Yuan T, Yang T, Chen H, Fu D, Hu Y, Wang J, et al. New insights into oxidative stress and inflammation during diabetes mellitusaccelerated atherosclerosis. Redox Biology. 2019; 20: 247-260.

[9] Alzamil H. Elevated Serum TNF- $\alpha$ is Related to Obesity in Type 2 Diabetes Mellitus and is Associated with Glycemic Control and Insulin Resistance. Journal of Obesity. 2020; 2020: 5076858 .

[10] Tsalamandris S, Antonopoulos AS, Oikonomou E, Papamikroulis G, Vogiatzi G, Papaioannou S, et al. The Role of Inflammation in Diabetes: Current Concepts and Future Perspectives. European Cardiology. 2019; 14: 50-59.

[11] Singh B, Singh JP, Kaur A, Singh N. Phenolic compounds as beneficial phytochemicals in pomegranate (Punica granatum L.) peel: a review. Food Chemistry. 2018; 261: 75-86.

[12] Viladomiu M, Hontecillas R, Lu P, Bassaganya-Riera J. Preventive and prophylactic mechanisms of action of pomegranate bioactive constituents. Evidence-Based Complementary and Alternative Medicine. 2013; 2013: 789764.

[13] Kandylis P, Kokkinomagoulos E. Food Applications and Potential Health Benefits of Pomegranate and its Derivatives. Foods. 2020; 9: 122.

[14] Vučić V, Grabež M, Trchounian A, Arsić A. Composition and Potential Health Benefits of Pomegranate: a Review. Current Pharmaceutical Design. 2019; 25: 1817-1827.

[15] Derakhshan Z, Ferrante M, Tadi M, Ansari F, Heydari A, Hosseini MS, et al. Antioxidant activity and total phenolic content of ethanolic extract of pomegranate peels, juice and seeds. Food and Chemical Toxicology. 2018; 114: 108-111.

[16] Gullón P, Astray G, Gullón B, Tomasevic I, Lorenzo JM. Pomegranate Peel as Suitable Source of High-Added Value Bioactives: Tailored Functionalized Meat Products. Molecules. 2020; 25: 2859

[17] Doostan F, Vafafar R, Zakeri-Milani P, Pouri A, Amini Afshar R, Mesgari Abbasi M. Effects of Pomegranate (Punica Granatum L.) Seed and Peel Methanolic Extracts on Oxidative Stress and Lipid Profile Changes Induced by Methotrexate in Rats. Advanced Pharmaceutical Bulletin. 2017; 7: 269-274.

[18] Dos Santos RL, Dellacqua LO, Delgado NTB, Rouver WN, Podratz PL, Lima LCF, et al. Pomegranate peel extract attenuates oxidative stress by decreasing coronary angiotensin-converting enzyme (ACE) activity in hypertensive female rats. Journal of
Toxicology and Environmental Health-Part A. 2016; 79: 998 1007.

[19] El-Hadary AE, Ramadan MF. Phenolic profiles, antihyperglycemic, antihyperlipidemic, and antioxidant properties of pomegranate (Punica granatum) peel extract. Journal of Food Biochemistry. 2019; 43: e12803.

[20] Grabež M, Škrbić R, Stojiljković M, Rudić-Grujić V, Šavikin $\mathrm{K}$, Menković $\mathrm{N}$, et al. Beneficial effects of pomegranate peel extract treatment on anthropometry and body composition of overweight patients with diabetes mellitus type-2: a randomised clinical trial. Scripta Medica. 2020; 51: 21-27.

[21] Grabež M, Škrbić R, Stojiljković MP, Rudić-Grujić V, Paunović $\mathrm{M}$, Arsić A, et al. Beneficial effects of pomegranate peel extract on plasma lipid profile, fatty acids levels and blood pressure in patients with diabetes mellitus type-2: a randomized, doubleblind, placebo-controlled study. Journal of Functional Foods. 2020; 64: 103692.

[22] Council of Europe. Tannins in herbal drugs. European Pharmacopoeia Commission. European Pharmacopoeia (pp. 275-276). 8th edn. Council of Europe: Strasbourg Cedex, France. 2013.

[23] World Health Organization. Pericarpium Granati. World Health Organization. WHO monographs on selected medicinal plants (pp. 117-126). WHO Press: Geneva, Switzerland. 2009.

[24] Gurinović M, Milešević J, Kadvan A, Djekić-Ivanković M, Debeljak-Martačić J, Takić M, et al. Establishment and advances in the online Serbian food and recipe data base harmonized with EuroFIRTM standards. Food Chemistry. 2016; 193: 30-38.

[25] Vasiljevic D, Veselinovic M, Jovanovic M, Jeremic N, Arsic A, Vucic V, et al. Evaluation of the effects of different supplementation on oxidative status in patients with rheumatoid arthritis. Clinical Rheumatology. 2016; 35: 1909-1915.

[26] Green LC, Wagner DA, Glogowski J, Skipper PL, Wishnok JS Tannenbaum SR. Analysis of nitrate, nitrite, and $[15 \mathrm{~N}]$ nitrate in biological fluids. Analytical Biochemistry. 1982; 126: 131-138.

[27] AuClair C, Voison E. Nitro bluetetrazolium reduction. In Greenwald RA (ed.) Handbook of Methods for Oxygen Radical Research (pp. 123-132). CRC Press: Boca Raton, FL. 1985.

[28] Pick E, Keisari Y. A simple colorimetric method for the measurement of hydrogen peroxide produced by cells in culture. Journal of Immunological Methods. 1980; 38: 161-170.

[29] Nikolic Turnic TR, Jakovljevic VL, Djuric DM, Jeremic NS, Jeremic JN, Milosavljevic IM, et al. Efficiency of atorvastatin and simvastatin in improving cardiac function during the different degrees of hyperhomocysteinemia. Canadian Journal of Physiology and Pharmacology. 2018; 96: 1040-1049.

[30] Marino M, Del Bo' C, Martini D, Porrini M, Riso P. A Review of Registered Clinical Trials on Dietary (Poly)Phenols: Past Efforts and Possible Future Directions. Foods. 2020; 9: 1606.

[31] de Souza Bastos A, Graves DT, de Melo Loureiro AP, Júnior CR, Corbi SCT, Frizzera F, et al. Diabetes and increased lipid peroxidation are associated with systemic inflammation even in well-controlled patients. Journal of Diabetes and its Complications. 2016; 30: 1593-1599.

[32] Mata-Cases M, Rodríguez-Sánchez B, Mauricio D, Real J, Vlacho B, Franch-Nadal J, et al. The Association between Poor Glycemic Control and Health Care Costs in People with Diabetes: a Population-Based Study. Diabetes Care. 2020; 43: 751758.

[33] Blahova J, Martiniakova M, Babikova M, Kovacova V, Mondockova V, Omelka R. Pharmaceutical Drugs and Natural Therapeutic Products for the Treatment of Type 2 Diabetes Mellitus. Pharmaceuticals. 2021; 14: 806

[34] Ma C, Chiua, YC, Wu C, Jou I-, Tu Y, Hung C, et al. Homocysteine causes dysfunction of chondrocytes and oxidative stress through repression of SIRT1/AMPK pathway: a possible link between hyperhomocysteinemia and osteoarthritis. Redox Biol- 
ogy. 2018; 15: 504-512.

[35] Malaguarnera G, Gagliano C, Giordano M, Salomone S, Vacante $\mathrm{M}$, Bucolo $\mathrm{C}$, et al. Homocysteine serum levels in diabetic patients with non proliferative, proliferative and without retinopathy. BioMed Research International. 2014; 2014: 191497.

[36] Huang T, Ren J, Huang J, Li D. Association of homocysteine with type 2 diabetes: a meta-analysis implementing Mendelian randomization approach. BMC Genomics. 2013; 14: 867.

[37] Li J, Zhang H, Shi M, Yan L, Xie M. Homocysteine is linked to macular edema in type 2 diabetes. Current Eye Research. 2014; 39: 730-735.

[38] Kumar J, Ingelsson E, Lind L, Fall T. No Evidence of a Causal Relationship between Plasma Homocysteine and Type 2 Diabetes: a Mendelian Randomization Study. Frontiers in Cardiovascular Medicine. 2015; 2: 11.

[39] Platt DE, Hariri E, Salameh P, Merhi M, Sabbah N, Helou M, et al. Type II diabetes mellitus and hyperhomocysteinemia: a complex interaction. Diabetology and Metabolic Syndrome. 2017; 9: 19.

[40] Wald DS, Law M, Morris JK. Homocysteine and cardiovascular disease: evidence on causality from a meta-analysis. BMJBritish Medical Journal. 2002; 325: 1202.

[41] Guo C, Wei J, Yang J, Xu J, Pang W, Jiang Y. Pomegranate juice is potentially better than apple juice in improving antioxidant function in elderly subjects. Nutrition Research. 2008; 28: 72 77.

[42] Petrovic S, Arsic A, Glibetic M, Cikiriz N, Jakovljevic V, Vucic V. The effects of polyphenol-rich chokeberry juice on fatty acid profiles and lipid peroxidation of active handball players: results from a randomized, double-blind, placebo-controlled study. Canadian Journal of Physiology and Pharmacology. 2016; 94: 1058-1063.

[43] Esmaeilinezhad Z, Barati-Boldaji R, Brett NR, de Zepetnek JOT, Bellissimo N, Babajafari S, et al. The effect of synbiotics pomegranate juice on cardiovascular risk factors in PCOS patients: a randomized, triple-blinded, controlled trial. Journal of Endocrinological Investigation. 2020; 43: 539-548.

[44] Sohrab G, Angoorani P, Tohidi M, Tabibi H, Kimiagar M, Nasrollahzadeh J. Pomegranate (Punicagranatum) juice decreases lipid peroxidation, but has no effect on plasma advanced glycated end-products in adults with type 2 diabetes: a randomized double-blind clinical trial. Food \& Nutrition Research. 2015; 59: 28551.

[45] Adela R, Nethi SK, Bagul PK, Barui AK, Mattapally S, Kuncha $\mathrm{M}$, et al. Hyperglycaemia enhances nitric oxide production in diabetes: a study from South Indian patients. PLoS ONE. 2015; 10: e0125270.

[46] Schneider MP, Ott C, Schmidt S, Kistner I, Friedrich S, Schmieder RE. Poor glycemic control is related to increased nitric oxide activity within the renal circulation of patients with type 2 diabetes. Diabetes Care. 2013; 36: 4071-4075.

[47] Alkathiri B, El-Khadragy MF, Metwally DM, Al-Olayan EM, Bakhrebah MA, Abdel Moneim AE. Pomegranate (Punica granatum) Juice Shows Antioxidant Activity against Cutaneous Leishmaniasis-Induced Oxidative Stress in Female BALB/c Mice. International Journal of Environmental Research and Public Health. 2017; 14: 1592.

[48] Sancaktutar AA, Bodakci MN, Hatipoglu NK, Soylemez H, Basarılı K, Turkcu G. The protective effects of pomegranate extracts against renal ischemia-reperfusion injury in male rats. Urology Annals. 2014; 6: 46-50.

[49] Boaz M, Smetana S, Weinstein T, Matas Z, Gafter U, Iaina A, et al. Secondary prevention with antioxidants of cardiovascular disease in endstage renal disease (SPACE): randomised placebocontrolled trial. Lancet. 2000; 356: 1213-1218.

[50] Yusuf S, Dagenais G, Pogue J, Bosch J, Sleight P. Vitamin E supplementation and cardiovascular events in high-risk patients The New England Journal of Medicine. 2000; 342: 154-160.

[51] Yamagata K. Polyphenols Regulate Endothelial Functions and Reducethe Risk of Cardiovasculare Disease Current Pharmaceutical Design. 2019; 25: 2443-2458.

[52] Del Bo' C, Bernardi S, Marino M, Porrini M, Tucci S, Guglielmetti A, et al. Systematic Review on Polyphenol Intake and Health Outcomes: Is there Sufficient Evidence to Define a Health-Promoting Polyphenol-Rich Dietary Pattern? Nutrients. 2019; 11: 1355.

[53] Stelmach-Mardas M, Kleiser C, Uzhova I, Peñalvo JL, La Torre G, Palys W, et al. Seasonality of food groups and total energy intake: a systematic review and meta-analysis. European Journal of Clinical Nutrition. 2016; 70: 700-708.

[54] Hosseini B, Saedisomeolia A, Wood LG, Yaseri M, Tavasoli S Effects of pomegranate extract supplementation on inflammation in overweight and obese individuals: a randomized controlled clinical trial. Complementary Therapies in Clinical Practice. 2016; 22: 44-50.

[55] Asgary S, Sahebkar A, Afshani MR, Keshvari M, Haghjooyjavanmard S, Rafieian-Kopaei M. Clinical evaluation of blood pressure lowering, endothelial function improving, hypolipidemic and anti-inflammatory effects of pomegranate juice in hypertensive subjects. Phytotherapy Research. 2014; 28: 193-199.

[56] Esmaillzadeh A, Tahbaz F, Gaieni I, Alavi-Majd H, Azadbakht L. Concentrated pomegranate juice improves lipid profiles in diabetic patients with hyperlipidemia. Journal of Medicinal Food. 2004; 7: 305-308.

[57] Kutan Fenercioglu A, Saler T, Genc E, Sabuncu H, Altuntas Y. The effects of polyphenol-containing antioxidants on oxidative stress and lipid peroxidation in Type 2 diabetes mellitus without complications. Journal of Endocrinological Investigation. 2010; 33: $118-124$.

[58] Hamoud S, Hayek T, Volkova N, Attias J, Moscoviz D, Rosenblat M, et al. Pomegranate extract (POMx) decreases the atherogenicity of serum and of human monocyte-derived macrophages (HMDM) in simvastatin-treated hypercholesterolemic patients: a double-blinded, placebo-controlled, randomized, prospective pilot study. Atherosclerosis. 2014; 232: 204-210.

[59] Mirmiran P, Fazeli MR, Asghari G, Shafiee A, Azizi F. Effect of pomegranate seed oil on hyperlipidaemic subjects: a doubleblind placebo-controlled clinical trial. The British Journal of $\mathrm{Nu}-$ trition. 2010; 104: 402-406.

[60] Manthou E, Georgakouli K, Deli CK, Sotiropoulos A, Fatouros IG, Kouretas D, et al. Effect of pomegranate juice consumption on biochemical parameters and complete blood count. Experimental and Therapeutic Medicine. 2017; 14: 1756-1762.

[61] Sohrab G, Roshan H, Ebrahimof S, Nikpayam O, Sotoudeh G, Siasi F. Effects of pomegranate juice consumption on blood pressure and lipid profile in patients with type 2 diabetes: a single-blind randomized clinical trial. Clinical Nutrition ESPEN. 2019; 29: 30-35.

[62] Sahebkar A, Simental-Mendía LE, Giorgini P, Ferri C, Grassi D. Lipid profile changes after pomegranate consumption: a systematic review and meta-analysis of randomized controlled trials. Phytomedicine. 2016; 23: 1103-1112.

[63] Lv O, Wang L, Li J, Ma Q, Zhao W. Effects of pomegranate peel polyphenols on lipid accumulation and cholesterol metabolic transformation in L-02 human hepatic cells via the PPAR $\gamma$ ABCA1/CYP7A1 pathway. Food \& Function. 2016; 7: 49764983.

[64] Hou C, Zhang W, Li J, Du L, Lv O, Zhao S, et al. Beneficial Effects of Pomegranate on Lipid Metabolism in Metabolic Disorders. Molecular Nutrition \& Food Research. 2019; 63: 1800773.

[65] Al-Rawi NH. Oxidative stress, antioxidant status and lipid profile in the saliva of type 2 diabetics. Diabetes and Vascular Dis- 
ease Research. 2011; 8: 22-28.

[66] Park K, Ahn CW, Lee SB, Kang S, Nam JS, Lee BK, et al. Elevated TyG Index Predict Progression of Coronary Artery Calcification. Diabetes Care. 2019; 42: 1569-1573.

[67] Wang L, Cong H, Zhang J, Hu Y, Wei A, Zhang Y, et al. Triglyceride-glucose index predicts adverse cardiovascular events in patients with diabetes and acute coronary syndrome. Cardiovascular Diabetology. 2021; 19: 80.

[68] Phosat C, Panprathip P, Chumpathat N, Prangthip P, Chantratita $\mathrm{N}$, Soonthornworasiri N, et al. Elevated C-reactive protein, interleukin 6, tumor necrosis factor alpha and glycemic load associated with type 2 diabetes mellitus in rural Thais: a crosssectional study. BMC Endocrine Disorders. 2017; 17: 44.

[69] Akbari M, Hassan-Zadeh V. IL-6 signalling pathways and the development of type 2 diabetes. Inflammopharmacology. 2018; 26: 685-698.

[70] Bashir H, Ahmad Bhat S, Majid S, Hamid R, Koul RK, Rehman MU, et al. Role of Inflammatory Mediators (TNF- $\alpha$, IL-6, CRP), Biochemical and Hematological Parameters in Type 2 Diabetes Mellitus Patients of Kashmir, India. Medical Journal of the Islamic Republic of Iran. 2020; 34: 5.

[71] Martín-Timón I, Sevillano-Collantes C, Segura-Galindo A, Del Cañizo-Gómez FJ. Type 2 diabetes and cardiovascular disease: have all risk factors the same strength? World Journal of Diabetes. 2014; 5: 444-470.

[72] Sohrab G, Nasrollahzadeh J, Zand H, Amiri Z, Tohidi M, Kimiagar M. Effects of Pomegranate Juice Consumption on Inflammatory Markers in Patients with Type 2 Diabetes: A Randomized, Placebo-Controlled Trial. Journal of Research in Medical
Sciences. 2014; 19: 215-220.

[73] Barati Boldaji R, Akhlaghi M, Sagheb MM, Esmaeilinezhad Z. Pomegranate juice improves cardiometabolic risk factors, biomarkers of oxidative stress and inflammation in hemodialysis patients: a randomized crossover trial. Journal of the Science of Food and Agriculture. 2020; 100: 846-854.

[74] Karwasra R, Singh S, Sharma D, Sharma S, Sharma N, Khanna $\mathrm{K}$. Pomegranate supplementation attenuates inflammation, joint dysfunction via inhibition of NF- $\mathrm{kB}$ signaling pathway in experimental models of rheumatoid arthritis. Journal of Food Biochemistry. 2019; 43: e12959.

[75] Mastrogiovanni F, Mukhopadhya A, Lacetera N, Ryan MT, Romani A, Bernini R, et al. Anti-Inflammatory Effects of Pomegranate Peel Extracts on in Vitro Human Intestinal Caco-2 Cells and Ex Vivo Porcine Colonic Tissue Explants. Nutrients. 2019; 11: 548.

[76] Du L, Li J, Zhang X, Wang L, Zhang W, Yang M, et al. Pomegranate Peel Polyphenols Inhibits Inflammation in LPS-Induced RAW264.7 Macrophages via the Suppression of TLR4/NF-KB Pathway Activation. Food and Nutrition Research. 2019; 63 .

[77] Xu J, Zhao Y, Aisa HA. Anti-inflammatory effect of pomegranate flower in lipopolysaccharide (LPS)-stimulated RAW264.7 macrophages. Pharmaceutical Biology. 2017; 55: 2095-2101.

[78] Mandal A, Bhatia D, Bishayee A. Anti-Inflammatory Mechanism Involved in Pomegranate-Mediated Prevention of Breast Cancer: the Role of NF-KB and Nrf2 Signaling Pathways. Nutrients. 2017; 9: 436 . 